\section{Adaptação transcultural: tradução e validação de conteúdo para o idioma português do modelo da Tripartite Influence Scale de insatisfação corporal}

\author{
Cross-cultural adaptation: translation and \\ Portuguese language content validation \\ of the Tripartite Influence Scale for \\ body dissatisfaction
}

\author{
1 Faculdade de Medicina, \\ Universidade de São Paulo, \\ São Paulo, Brasil. \\ 2 Departamento de Ciências \\ da Saúde, Universidad \\ Federal de São Paulo, \\ Santos, Brasil. \\ 3 School of Medicine \\ University of California, \\ San Francisco, U.S.A. \\ Correspondência \\ M. A. Conti \\ Ambulatório de Bulimia e \\ Transtornos Alimentares, \\ Instituto de Psiquiatria, \\ Hospital das Clínicas, \\ Faculdade de Medicina, \\ Universidade de São Paulo. \\ Rua Dr. Ovídio Pires de \\ Campos 785, 2o andar, \\ São Paulo, SP \\ 05403-010, Brasil. \\ maconti@usp.br
}

\begin{abstract}
The aim of this study was to translate and adapt the Tripartite Influence Scale to the Portuguese language and evaluate its content validity and internal consistency. Six steps included: (1) translation; (2) back-translation; (3) technique revision and semantic evaluation; (4) conduct validation by professional experts (judges); (5) assessment of comprehensibility by the target population, using a verbal rating scale; and (6) evaluation of the internal consistency using Cronbach's alpha coefficient. The 43 questions were translated and adapted to the Portuguese language. The final version consisted of 39 items, with content validity for three constructs (media, family, and friends), clarity and easy understanding, and good internal agreement (Cronbach's alpha coefficients $>0.80$ ). The instrument was successfully translated and adapted to Portuguese and showed good content validity, verbal comprehensibility, and internal consistency. Further analysis of external validity, equivalence of measurement, and reproducibility are necessary.
\end{abstract}

Body Image; Eating Disorders; Adolescent

\author{
Maria Aparecida Conti 1 \\ Fernanda Scagliusi 2 \\ Gisele Kawamura de Oliveira Queiroz ${ }^{1}$ \\ Norman Hearst 3 \\ Táki Athanássios Cordás ${ }^{1}$
}

\section{Introdução}

Os transtornos alimentares configuram-se como síndromes crônicas de difícil diagnóstico e tratamento ${ }^{1}$. Esforços são direcionados para o entendimento dos fatores que desempenham um papel no desenvolvimento e na manutenção destes transtornos, bem como em relação aos distúrbios da imagem corporal 2 , aspecto este relevante na psicopatologia destes quadros.

Fatores relacionados a aspectos biológicos, sociais e interpessoais são pesquisados como potenciais fatores de risco ${ }^{3}$. Aspectos como baixa auto-estima, relação afetiva pobre com a família, pressão familiar, dietas, insatisfação corporal 4, abuso sexual, interesse pelo peso e corpo 5 são citados igualmente como potenciais fatores de risco, além do retorno verbal negativo, como por exemplo frases que depreciam e desvalorizam o indivíduo, bullying e a internalização do modelo ideal proposto pela mídia 6 .

As pesquisas relacionadas aos fatores de risco têm se desenvolvido na última década, no entanto, carecem de modelos teóricos para orientar a seleção das medidas mais adequadas e auxiliar no teste de hipóteses 7 .

Um modelo existente refere-se ao The Tripartite Influence Model of Body Dissatisfaction and Eating Disturbance, que incorpora algumas variáveis que afetam o desenvolvimento de distúrbios de imagem corporal e de transtornos alimentares, mais especificamente para comportamento 
restritivos, no caso da anorexia nervosa e comportamentos bulímicos 8. É composto por três fontes primárias de influências - amigos, família e mídia -, partindo-se do princípio que a manifestação do seu efeito para a imagem corporal e para os transtornos alimentares se dá por meio de dois mecanismos primários: comparação da aparência das jovens entre si e a internalização do modelo ideal de magreza 9 .

No estudo original os autores aplicaram um modelo de estrutura de co-variância examinando nove variáveis latentes: influências familiares, influência da mídia, influências dos colegas, processo de comparação social, perfeccionismo, funcionamento psicológico global, insatisfação corporal, comportamentos alimentares restritivos e comportamentos bulímicos. Comprovaram a presença dos dois mecanismos (comparação da aparência e a internalização do modelo de magreza) como mediadores para as três fontes primárias de influência na insatisfação corporal e transtornos alimentares 9 .

Keery et al. 7 avaliaram este modelo teórico e propuseram uma escala que abrangesse as três fontes de influência formativa (amigos, família e mídia) que afetam a imagem corporal e o desenvolvimento de transtornos alimentares nas adolescentes e jovens. Comprovaram a adequação do modelo e do instrumento propostos como ferramentas profícuas para o entendimento do processo que podem predispor jovens mulheres a desenvolver distúrbios de imagem corporal e transtornos alimentares.

Esta escala - Tripatite Influence Scale-é composta de 43 itens de autopreenchimento, com as respostas na forma likert de pontos tratando as questões por meio do interesse, freqüência, pressão, preocupação e ênfase dada pelo sujeito. O escore é calculado pela soma das respostas e quanto menor a pontuação maior o comprometimento. Este instrumento ainda não possui sua versão em português e não foram desenvolvidos estudos para verificar as evidências de validade para a realidade brasileira. A sua vantagem é concentrar informações relativas a diversas fontes de influência em um único instrumento, ser auto-aplicável, o que facilita sua aplicação em grandes populações, além de oferecer dados referentes aos temas estudados. Este trabalho objetiva descrever seu processo de adaptação transcultural, que integra a tradução, retro-tradução, validação de conteúdo e avaliação de sua consistência interna.

\section{Métodos}

O processo de adaptação transcultural baseou-se nos procedimentos sugeridos por Pasquali $10 \mathrm{e}$ Herdman et al. 11 e aplicados por Moraes et al. 12 e Conti et al. 13 .

\section{Procedimentos}

Foram cumpridas seis etapas, a saber: primeira tradução do instrumento original em inglês para a língua portuguesa; segunda - retro-tradução do instrumento em português para a língua inglesa; terceira - revisão técnica e avaliação da equivalência semântica; quarta - validação do conteúdo; quinta - avaliação do instrumento pelos especialistas e população-alvo; sexta - avaliação da consistência interna do instrumento.

A primeira etapa consistiu na tradução do instrumento original do idioma inglês para o português, feita independentemente por dois pesquisadores experientes e fluentes em inglês. Para a segunda etapa, as versões (tradução 1 e 2) foram novamente traduzidas para o inglês, por um professor nativo de língua inglesa. A etapa seguinte referiu-se à revisão técnica e avaliação da equivalência semântica das versões, desenvolvida por dois profissionais (um psicólogo e um nutricionista) especialistas na área de adaptação de escalas. Duas questões foram priorizadas na avaliação da equivalência semântica: o significado referencial (denotativo) do instrumento, na avaliação da versão traduzida (português) em relação à versão final (inglês) e o significado geral (conotativo), na comparação do instrumento original em relação à versão final (inglês), com o intuito de se garantir a transferência dos significados das palavras entre os dois idiomas 14 Foram realizados ajustes e uma versão corrigida do instrumento foi elaborada.

Na quarta etapa, para avaliar a validade de conteúdo, a versão corrigida do instrumento foi apresentada a dez profissionais especialistas da área de transtornos alimentares. Foi solicitado que lessem a versão traduzida do instrumento atentamente e identificassem a qual fator cada item pertencia (amigos, familiares e mídia). Foi utilizada como critério de adequação uma taxa de acerto de pelo menos $80 \%$ para cada questão 10 .

Para avaliação da clareza e do grau de compreensão de cada questão e do instrumento em sua íntegra foi solicitado, seqüencialmente, aos mesmos profissionais, que respondessem uma escala verbal-numérica adaptada 13,15. Foram orientados para responder a seguinte questão: “Você entendeu o que foi perguntado?". As respostas eram do tipo escala likert: 0 (não entendi nada); 1 (entendi só um pouco); 2 (entendi mais 
ou menos); 3 (entendi quase tudo, mas tive algumas dúvidas); 4 (entendi quase tudo); 5 (entendi perfeitamente e não tenho dúvidas). Conforme Conti et al. 13 foi estabelecido que as respostas 0 , 1, 2 e 3 seriam consideradas como indicadores de uma compreensão insuficiente. Foi solicitado ainda, caso o especialista não compreendesse a questão ou a linguagem não parecesse adequada, que sugerisse alterações, justificando os motivos. Uma nova versão do instrumento foi formulada a partir destes dados.

Na etapa seguinte, a versão final foi apresentada a 108 jovens, de ambos os sexos, estudantes do Ensino Superior do curso de Administração. Os jovens foram selecionados de forma aleatória simples e concordaram em participar voluntariamente. Para metade do grupo (55 jovens) o instrumento foi aplicado na apresentação de uma escala verbal-numérica adaptada 13, exatamente da mesma forma como aplicada aos especialistas, seguindo os procedimentos metodológicos descritos anteriormente. Almejou-se avaliar o grau de compreensão de cada questão e do instrumento em sua íntegra. Ao término do preenchimento da escala foi solicitado a este jovem que registrasse suas sugestões, caso percebesse alguma dificuldade para a compreensão nos itens que compõem o instrumento.

Para a última etapa, a versão final do instrumento, Escala de Influência dos Três Fatores (Tabela 1), foi aplicada ao restante do grupo (53 jovens), na forma likert de pontos, de acordo com a seguinte instrução: "Responda este questionário seguindo a escala abaixo”. As opções de respostas foram: 1 (sempre); 2 (quase sempre); 3 (freqüentemente); 4 (algumas vezes); 5 (nunca). Com esse material foi possível verificar o grau de consistência interna das questões que compõem a escala, por meio do coeficiente alfa de Cronbach.

Para as análises estatísticas utilizou-se o programa SPSS versão 15.0 (SPSS Inc., Chicago, Estados Unidos). Desenvolveram-se análises descritiva (média, desvios-padrão - DP, valores mínimos e máximos, freqüências) e inferencial dos dados por meio do cálculo do coeficiente alfa de Cronbach.

O presente estudo está de acordo com as normas da Portaria $n^{\circ}$. 196/96 do Conselho Nacional de Saúde e foi aprovado pelo Comitê de Ética e Pesquisa do Hospital das Clínicas da Faculdade de Medicina da Universidade de São Paulo (protocolo: 0586/08).

\section{Resultados}

Participaram do estudo dez profissionais especialistas da área de transtornos alimentares (três psicólogos, três nutricionistas, um educador físico, um enfermeiro e dois psiquiatras) e 108 estudantes, sendo 51 ( 18 anos e DP $=2,3$ anos) e 57 participantes (19 anos e DP $=3,2$ anos) do sexo masculino e feminino, respectivamente.

\section{Tradução e adaptação cultural}

Na tradução do instrumento houve necessidade de adaptação da palavra fitness e da expressão skip meals que na versão final ficaram como "boa forma” e "pular refeição", respectivamente. As questões 11 e 13 exigiram certa atenção. Na versão original (Tabela 2) expressam duas condições (o quanto você pesa; ser e tornar-se gorda). Como na tradução literal os conteúdos ficaram repetitivos, adaptou-se a frase em uma única condição (está ou pode se tornar gorda) para garantir maior clareza

Optou-se pela substituição do pronome "meu" para "seu" nas questões de conteúdo familiar com o intuito de garantir uma maior compreensão das mesmas. Nas questões $21 \mathrm{e}$ 22 utilizou-se o tempo verbal no presente "faz comentários ou te provoca sobre tua aparência", conforme descrito nas Tabelas 2 e 3.

Houve necessidade de se introduzir a expressão “o quanto eles pesam" na questão 26 , substituindo "o peso deles" e os pronomes "ele, eles e ela" na frase "que eles sejam tão magros quanto possível" das questões 16, 19 e 30, respectivamente (Tabelas 2 e 3 ).

Quatro questões foram excluídas do instrumento original: 27, 30, 39 e 43 (Tabela 2). As duas primeiras questionam a freqüência de preocupação dos pais quanto ao consumo, no entanto, não fica claro se diz respeito à quantidade, ou seja, ao consumo energético ou à qualidade, ou seja, em relação ao tipo de alimento. Já as duas questões restantes abordam temas que se repetem (importância de peso e forma corporais), já explorados nas questões 17, 20 e 31 do instrumento final.

Outro ponto diz respeito à adaptação do instrumento para a população masculina. Em todas as questões foram incluídas a extensão “(a)” para garantir a compreensão de ambos os sexos.

As etapas de tradução, retro-tradução e versão final podem ser observadas nas Tabelas 2 e 3.

\section{Validação de conteúdo}

Houve unanimidade entre os especialistas para os três conteúdos que compõem o instrumento: 
Escala de Influência dos Três Fatores (mídia, família e amigos).

\section{Questões}

1. As revistas que eu leio e os programas de TV que assisto enfatizam que é importante ser magro(a).

2. As revistas que leio e os programas de TV que assisto enfatizam a importância da aparência (forma corporal, peso, roupas).

3. As revistas que leio e os programas de TV que assisto enfatizam a prática de dietas para perder peso.

4. Eu tenho sentido pressão da mídia para perder peso.

5. Eu me interessaria em assistir um novo programa de TV se o tema fosse dieta.

6. Eu me interessaria em assistir um novo programa de TV se o tema fosse boa forma e exercícios.

7. Eu me interessaria em assistir um novo programa de TV se o tema fosse moda.

8. Eu me interessaria em ler uma nova revista se o tema fosse prática de dieta.

9. Eu me interessaria em ler uma nova revista se os temas fossem boa forma e exercícios.

10. Eu me interessaria em ler uma nova revista se o tema fosse moda.

11. O quanto a sua mãe é preocupada se você está ou pode se tornar muito gordo(a)?

12. Quão importante é para sua mãe que você seja magro(a)?

13. O quanto o seu pai é preocupado se você está ou pode se tornar muito gordo(a)?

14. Quão importante é para seu pai que você seja magro(a)?

15. Seu pai está fazendo dieta para perder peso.

16. É importante para o seu pai que ele seja tão magro quanto possível.

17. A aparência física do seu pai (forma corporal, peso, roupas) é importante para ele.

18. Sua mãe está fazendo dieta para perder peso.

19. É importante para a sua mãe que ela seja tão magra quanto possível.

20. A aparência física de sua mãe (forma corporal, peso, roupas) é importante para ela.

21. Seu pai faz comentários ou te provoca sobre sua aparência.

22. Sua mãe faz comentários ou te provoca sobre sua aparência.

23. Com que freqüência seus pais comentam sobre os pesos um do outro?

24. Com que freqüência seus pais encorajam um ao outro a perder peso?

25. Com que freqüência seus pais conversam sobre peso e prática de dieta?

26. Com que freqüência seus pais se preocupam sobre o quanto eles pesam?

27. Com que freqüência seus pais fazem dietas?

28. Você acha que seus pais reparam muito no peso e formas corporais um do outro?

29. Um ou mais de meus amigo(a)s e colegas de classe estão fazendo dieta para perder peso.

30. É importante para meus amigo(a)s e colegas de classe que eles sejam tão magro(a)s quanto possível.

31. A aparência física dos meus (minhas) amigo(a)s e colegas de classe (forma corporal, peso, roupas) é importante para eles.

32. Seus amigo(a)s e colegas de classe fazem comentários ou te provocam sobre sua aparência

33. Com que freqüência seus amigo(a)s e colegas de classe comentam entre si sobre seus pesos?

34. Com que freqüência seus amigo(a)s e colegas de classe encorajam um ao outro a perder peso?

35. Com que freqüência seus amigo(a)s e colegas de classe conversam sobre peso ou prática de dietas?

36. Com que freqüência seus amigo(a)s e colegas de classe se preocupam sobre o quanto eles pesam?

37. Com que freqüência seus amigo(a)s e colegas de classe fazem dietas?

38. Com que freqüência seus amigo(a)s e colegas de classe pulam refeições?

39. Você acha que seus amigo(a)s e colegas de classe reparam muito no peso e formas corporais um do outro?

mídia, família e amigos, com um grau de concordância de $100 \%$ (Tabela 4).

\section{Compreensão verbal}

Para os especialistas as questões demonstraram ser de fácil compreensão, registrando-se valor médio superior a 4,6 (valor máximo = 5,0) O mesmo pode ser observado na população alvo, com valores médios superior a 4,6 (valor máximo = $5,0)$ (Tabela 4). 
Avaliação da equivalência semântica da escala original Tripartite Influence Scale e a versão traduzida para o português.

\begin{tabular}{|c|c|}
\hline Documento original & Versão traduzida \\
\hline Tripartite Influence Scale (media, parent, peers) & Escala de Influência dos Três Fatores (mídia, família e amigos) \\
\hline $\begin{array}{l}\text { 1. The magazines I read and TV shows I watch emphasize that it is } \\
\text { important to be thin. }\end{array}$ & $\begin{array}{l}\text { 1. As revistas que eu leio e os programas de TV que assisto enfatizam que é } \\
\text { importante ser magro. }\end{array}$ \\
\hline $\begin{array}{l}\text { 2. The magazines I read and the TV shows } 1 \text { watch emphasize the } \\
\text { importance of appearance (shape, weight, clothing). }\end{array}$ & $\begin{array}{l}\text { 2. As revistas que leio e os programas de TV que assisto enfatizam a } \\
\text { importância da aparência (forma corporal, peso, roupas). }\end{array}$ \\
\hline $\begin{array}{l}\text { 3. The magazines I read and TV shows I watch emphasize dieting to } \\
\text { lose weight. }\end{array}$ & $\begin{array}{l}\text { 3. As revistas que leio e os programas de TV que assisto enfatizam a prática } \\
\text { de dietas para perder peso. }\end{array}$ \\
\hline 4. I have felt pressure from the media to lose weight. & 4. Eu tenho sentido pressão da mídia para perder peso. \\
\hline $\begin{array}{l}\text { 5. I would be interested in watching a new TV show if the topic is } \\
\text { dieting. }\end{array}$ & $\begin{array}{l}\text { 5. Eu me interessaria em assistir um novo programa de TV se o tema fosse } \\
\text { dieta. }\end{array}$ \\
\hline $\begin{array}{l}\text { 6. I would be interested in watching a new TV show if the topic is } \\
\text { fitness and exercise. }\end{array}$ & $\begin{array}{l}\text { 6. Eu me interessaria em assistir um novo programa de TV se o tema fosse } \\
\text { boa forma e exercícios. }\end{array}$ \\
\hline $\begin{array}{l}\text { 7. I would be interested in watching a new TV show if the topic is } \\
\text { fashion. }\end{array}$ & $\begin{array}{l}\text { 7. Eu me interessaria em assistir um novo programa de TV se o tema fosse } \\
\text { moda. }\end{array}$ \\
\hline $\begin{array}{l}\text { 8. I would be interested in reading a new magazine if the topic is } \\
\text { dieting. }\end{array}$ & $\begin{array}{l}\text { 8. Eu me interessaria em ler uma nova revista se o tema fosse prática de } \\
\text { dieta. }\end{array}$ \\
\hline $\begin{array}{l}\text { 9. I would be interested in reading a new magazine if the topic is } \\
\text { fitness and exercise. }\end{array}$ & $\begin{array}{l}\text { 9. Eu me interessaria em ler uma nova revista se os temas fossem boa forma } \\
\text { e exercícios. }\end{array}$ \\
\hline $\begin{array}{l}\text { 10. I would be interested in reading a new magazine if the topic is } \\
\text { fashion. }\end{array}$ & 10. Eu me interessaria em ler uma nova revista se o tema fosse moda. \\
\hline $\begin{array}{l}\text { 11. How concerned is your mother about whether you weigh too } \\
\text { much or are too fat or might become too fat? }\end{array}$ & $\begin{array}{l}\text { 11. Quão preocupada é a sua mãe se você pesa muito ou é muito gorda ou } \\
\text { pode se tornar muito gorda? }\end{array}$ \\
\hline 12. How important is it to your mother that you be thin? & 12. Quão importante é para sua mãe que você seja magra? \\
\hline $\begin{array}{l}\text { 13. How concerned is your father about whether you weigh too } \\
\text { much or are too fat of might become too fat? }\end{array}$ & $\begin{array}{l}\text { 13. Quão preocupado é o seu pai se você pesa muito ou é muito gorda ou } \\
\text { pode se tornar muito gorda? }\end{array}$ \\
\hline 14. How important is it to your father that you be thin? & 14. Quão importante é para seu pai que você seja magra? \\
\hline 15. My father is on a diet to lose weight. & 15. Meu pai está fazendo dieta para perder peso. \\
\hline 16. It is important to my father that he be as thin as possible. & 16. É importante para o meu pai que ele seja tão magro quanto possível. \\
\hline $\begin{array}{l}\text { 17. My father's physical appearance (shape, weight, clothing) is } \\
\text { important to him. }\end{array}$ & $\begin{array}{l}\text { 17. A aparência física do meu pai (forma corporal, peso, roupas) é importante } \\
\text { para ele. }\end{array}$ \\
\hline 18. My mother is on a diet to lose weight. & 18. Minha mãe está fazendo dieta para perder peso. \\
\hline 19. It is important to my mother that she be as thin as possible. & 19. É importante para a minha mãe que ela seja tão magra quanto possível. \\
\hline $\begin{array}{l}\text { 20. My mother's physical appearance (shape, weight, clothing) is } \\
\text { important to her. }\end{array}$ & $\begin{array}{l}\text { 20. A aparência física da minha mãe (forma corporal, peso, roupas) é } \\
\text { importante para ela. }\end{array}$ \\
\hline $\begin{array}{l}\text { 21. Your father made comments or teased you about your } \\
\text { appearance. }\end{array}$ & 21. Seu pai fez comentários ou te provoca sobre tua aparência. \\
\hline $\begin{array}{l}\text { 22. Your mother made comments or teased you about your } \\
\text { appearance. }\end{array}$ & 22. Sua mãe fez comentários ou te provoca sobre tua aparência. \\
\hline 23. How often do your parents comment on each other's weight? & 23. Com que freqüência seus pais comentam sobre os pesos um do outro? \\
\hline $\begin{array}{l}\text { 24. How often do your parents encourage each other to lose } \\
\text { weight? }\end{array}$ & 24. Com que freqüência seus pais encorajam um ao outro a perder peso? \\
\hline 25. How often do your parents talk about weight or dieting? & 25. Com que freqüência seus pais conversam sobre peso e pratica de dieta? \\
\hline 26. How often do your parents worry about their weight? & 26. Com que freqüência seus pais se preocupam sobre o peso deles? \\
\hline 27. How often do your parents worry about what they eat? & 27. Com que freqüência seus pais se preocupam sobre o que eles comem? \\
\hline 28. How often do your parents diet? & 28. Com que freqüência seus pais fazem dietas? \\
\hline $\begin{array}{l}\text { 29. Do you think your parents take a lot of notice of each other's } \\
\text { weight and shape? }\end{array}$ & $\begin{array}{l}\text { 29. Você acha que seus pais reparam muito no peso e formas corporais um } \\
\text { do outro? }\end{array}$ \\
\hline 30. Are weight and shape important to your parents? & 30. Peso e formas corporais são importantes para os seus pais? \\
\hline $\begin{array}{l}\text { 31. One or more of my friends and classmates is on a diet to lose } \\
\text { weight. }\end{array}$ & $\begin{array}{l}\text { 31. Um ou mais de meus amigos e colegas de classe estão fazendo dieta } \\
\text { para perder peso. }\end{array}$ \\
\hline $\begin{array}{l}\text { 32. It is important to my friends and classmates that they be as thin } \\
\text { as possible. }\end{array}$ & $\begin{array}{l}\text { 32. É importante para meus amigos e colegas de classe que sejam tão } \\
\text { magros quanto possível. }\end{array}$ \\
\hline
\end{tabular}


Avaliação da equivalência semântica da escala original Tripartite Influence Scale e a versão traduzida para o português.

\begin{tabular}{|c|c|}
\hline Documento original & Versão traduzida \\
\hline Tripartite Influence Scale (media, parent, peers) & Escala de Influência dos Três Fatores (mídia, família e amigos) \\
\hline $\begin{array}{l}\text { 33. My friends and classmates' physical appearance (shape, weight, } \\
\text { clothing) is important to them. }\end{array}$ & $\begin{array}{l}\text { 33. A aparência física dos meus amigos e colegas de classe (forma corporal, } \\
\text { peso, roupas) é importante para eles. }\end{array}$ \\
\hline $\begin{array}{l}\text { 34. Your friends and classmates made comments or teased you } \\
\text { about your appearance. }\end{array}$ & $\begin{array}{l}\text { 34. Seus amigos e colegas de classe fazem comentários ou te provocam } \\
\text { sobre sua aparência. }\end{array}$ \\
\hline $\begin{array}{l}\text { 35. How often do your friends and classmates comment on each } \\
\text { other's weight? }\end{array}$ & $\begin{array}{l}\text { 35. Com que freqüência seus amigos e colegas de classe comentam entre si } \\
\text { sobre seus pesos? }\end{array}$ \\
\hline $\begin{array}{l}\text { 36. How often do your friends and classmates encourage each other } \\
\text { to lose weight? }\end{array}$ & $\begin{array}{l}\text { 36. Com que freqüência seus amigos e colegas de classe encorajam um ao } \\
\text { outro a perder peso? }\end{array}$ \\
\hline $\begin{array}{l}\text { 37. How often do your friends and classmates talk about weight or } \\
\text { dieting? }\end{array}$ & $\begin{array}{l}\text { 37. Com que freqüência seus amigos e colegas de classe conversam sobre } \\
\text { peso ou pratica de dietas? }\end{array}$ \\
\hline $\begin{array}{l}\text { 38. How often do your friends and classmates worry about their } \\
\text { weight? }\end{array}$ & $\begin{array}{l}\text { 38. Com que freqüência seus amigos e colegas de classe se preocupam } \\
\text { sobre seus pesos? }\end{array}$ \\
\hline $\begin{array}{l}\text { 39. How often do your friends and classmates worry what } \\
\text { they eat? }\end{array}$ & $\begin{array}{l}\text { 39. Com que freqüência seus amigos e colegas de classe se preocupam } \\
\text { sobre o que eles comem? }\end{array}$ \\
\hline 40. How often do your friends and classmates diet? & 40. Com que freqüência seus amigos e colegas de classe fazem dietas? \\
\hline 41. How often do your friends and classmates skip meals? & 41. Com que freqüência seus amigos e colegas de classe pulam refeições? \\
\hline $\begin{array}{l}\text { 42. Do you think that your friends and classmates take a lot of } \\
\text { notice in each other's weight and shape? }\end{array}$ & $\begin{array}{l}\text { 42. Você acha que seus amigos e colegas de classe reparam muito no peso e } \\
\text { formas corporais um do outro? }\end{array}$ \\
\hline 43. Are weight and shape important to your friends and classmates? & $\begin{array}{l}\text { 43. Peso e formas corporais são importantes para seus amigos e colegas de } \\
\text { classe? }\end{array}$ \\
\hline
\end{tabular}

Avaliação da equivalência semântica entre versão retraduzida e a versão final do Tripartite Influence Scale.

\begin{tabular}{|c|c|}
\hline Documento original & Versão traduzida \\
\hline Tripartite Influence Scale (media, parent, peers) & Escala de Influência dos Três Fatores (mídia, família e amigos) \\
\hline $\begin{array}{l}\text { 1. The magazines I read and television programs I see emphasize } \\
\text { the importance of being thin. }\end{array}$ & $\begin{array}{l}\text { 1. As revistas que eu leio e os programas de TV que assisto enfatizam que é } \\
\text { importante ser magro(a). }\end{array}$ \\
\hline $\begin{array}{l}\text { 2. The magazines I read and television programs I see emphasize } \\
\text { the importance of appearance (body shape, weight, clothing). }\end{array}$ & $\begin{array}{l}\text { 2. As revistas que leio e os programas de TV que assisto enfatizam a } \\
\text { importância da aparência (forma corporal, peso, roupas). }\end{array}$ \\
\hline $\begin{array}{l}\text { 3. The magazines I read and television programs I see emphasize } \\
\text { diets to lose weight. }\end{array}$ & $\begin{array}{l}\text { 3. As revistas que leio e os programas de TV que assisto enfatizam a prática } \\
\text { de dietas para perder peso. }\end{array}$ \\
\hline 4. I have felt pressure from the media to lose weight. & 4. Eu tenho sentido pressão da mídia para perder peso. \\
\hline $\begin{array}{l}5 . \text { I would be interested in watching a new TV program about } \\
\text { dieting. }\end{array}$ & $\begin{array}{l}\text { 5. Eu me interessaria em assistir um novo programa de TV se o tema fosse } \\
\text { dieta. }\end{array}$ \\
\hline $\begin{array}{l}\text { 6. I would be interested in watching a new TV program about } \\
\text { exercise and being in shape. }\end{array}$ & $\begin{array}{l}\text { 6. Eu me interessaria em assistir um novo programa de TV se o tema fosse } \\
\text { boa forma e exercícios. }\end{array}$ \\
\hline $\begin{array}{l}\text { 7. I would be interested in watching a new TV program about } \\
\text { fashion. }\end{array}$ & $\begin{array}{l}\text { 7. Eu me interessaria em assistir um novo programa de TV se o tema fosse } \\
\text { moda. }\end{array}$ \\
\hline 8. I would be interested in reading a new magazine about diets. & 8. Eu me interessaria em ler uma nova revista se o tema fosse prática de dieta. \\
\hline $\begin{array}{l}\text { 9. I would be interested in reading a new magazine about exercise } \\
\text { and being in shape. }\end{array}$ & $\begin{array}{l}\text { 9. Eu me interessaria em ler uma nova revista se os temas fossem boa forma } \\
\text { e exercícios. }\end{array}$ \\
\hline 10. I would be interested in reading a new magazine about fashion. & 10. Eu me interessaria em ler uma nova revista se o tema fosse moda. \\
\hline $\begin{array}{l}\text { 11. How worried is your mother that your weigh too much or are too } \\
\text { fat or could become too fat? }\end{array}$ & $\begin{array}{l}\text { 11. Quão preocupada é a sua mãe se você pesa muito ou é muito gordo(a) } \\
\text { ou pode se tornar muito gordo (a)? }\end{array}$ \\
\hline 12. How important is it to your mother that you be thin? & 12. Quão importante é para sua mãe que você seja magro(a)? \\
\hline $\begin{array}{l}\text { 13. How worried is your father that your weigh too much or are too } \\
\text { fat or could become too fat? }\end{array}$ & $\begin{array}{l}\text { 13. Quão preocupado é o seu pai se você pesa muito ou é muito gordo(a) ou } \\
\text { pode se tornar muito gordo(a)? }\end{array}$ \\
\hline
\end{tabular}

(continua) 
Avaliação da equivalência semântica entre versão retraduzida e a versão final do Tripartite Influence Scale.

\begin{tabular}{|c|c|}
\hline Documento original & Versão traduzida \\
\hline Tripartite Influence Scale (media, parent, peers) & Escala de Influência dos Três Fatores (mídia, família e amigos) \\
\hline 14. How important is it to your father that you be thin? & 14. Quão importante é para seu pai que você seja magro(a)? \\
\hline 15. My father is dieting to lose weight. & 15. Seu pai está fazendo dieta para perder peso. \\
\hline 16. It is important to my father to be as thin as possible. & 16. É importante para o seu pai que ele seja tão magro quanto possível. \\
\hline $\begin{array}{l}\text { 17. My father's physical appearance (body shape, weight, clothes) is } \\
\text { important to him. }\end{array}$ & $\begin{array}{l}\text { 17. A aparência física do seu pai (forma corporal, peso, roupas) é importante } \\
\text { para ele. }\end{array}$ \\
\hline 18. My mother is dieting to lose weight. & 18. Sua mãe está fazendo dieta para perder peso. \\
\hline 19. It is important to my mother to be as thin as possible. & 19. É importante para a sua mãe que ela seja tão magra quanto possível. \\
\hline $\begin{array}{l}\text { 20. My mother's physical appearance (shape, weight, clothes) is } \\
\text { important to her. }\end{array}$ & $\begin{array}{l}\text { 20. A aparência física de sua mãe (forma corporal, peso, roupas) é importante } \\
\text { para ela. }\end{array}$ \\
\hline 21. Your father made remarks or teased you about your appearance. & 21. Seu pai fez comentários ou te provoca sobre tua aparência. \\
\hline $\begin{array}{l}\text { 22. Your mother made remarks or teased you about your } \\
\text { appearance. }\end{array}$ & 22. Sua mãe fez comentários ou te provoca sobre tua aparência. \\
\hline 23. How often do your parents comment about each other's weight? & 23. Com que freqüência seus pais comentam sobre os pesos um do outro? \\
\hline $\begin{array}{l}\text { 24. How often do your parents encourage each other to lose } \\
\text { weight? }\end{array}$ & 24. Com que freqüência seus pais encorajam um ao outro a perder peso? \\
\hline 25. How often do your parents talk about weight or dieting? & 25. Com que freqüência seus pais conversam sobre peso e prática de dieta? \\
\hline 26. How often do your parents worry about their weight? & 26. Com que freqüência seus pais se preocupam sobre o quanto eles pesam? \\
\hline 27. How often do your parents diet? & 27. Com que freqüência seus pais fazem dietas? \\
\hline $\begin{array}{l}\text { 28. Do you think that your parents pay a lot of attention of each } \\
\text { other's body shape? }\end{array}$ & $\begin{array}{l}\text { 28. Você acha que seus pais reparam muito no peso e formas corporais um } \\
\text { do outro? }\end{array}$ \\
\hline $\begin{array}{l}\text { 29. One or more of my friends and classmates is dieting to lose } \\
\text { weight. }\end{array}$ & $\begin{array}{l}\text { 29. Um ou mais de meus amigo(a)s e colegas de classe estão fazendo dieta } \\
\text { para perder peso }\end{array}$ \\
\hline $\begin{array}{l}\text { 30. It is important to my friends and classmates to be as thin as } \\
\text { possible. }\end{array}$ & $\begin{array}{l}\text { 30. É importante para meus amigo(a)s e colegas de classe que sejam tão } \\
\text { magro(a)s quanto possível. }\end{array}$ \\
\hline $\begin{array}{l}\text { 31. The physical appearance of my friends and classmates (shape, } \\
\text { weight, clothing) is important to them. }\end{array}$ & $\begin{array}{l}\text { 31. A aparência física dos meus amigo(a)s e colegas de classe (forma } \\
\text { corporal, peso, roupas) é importante para eles. }\end{array}$ \\
\hline $\begin{array}{l}\text { 32. Your friends and classmates made remarks or teased you about } \\
\text { your appearance. }\end{array}$ & $\begin{array}{l}\text { 32. Seus amigo(a)s e colegas de classe fazem comentários ou te provocam } \\
\text { sobre sua aparência. }\end{array}$ \\
\hline $\begin{array}{l}\text { 33. How often do your friends and classmates make remarks or } \\
\text { teased to each other about their weight? }\end{array}$ & $\begin{array}{l}\text { 33. Com que freqüência seus amigo(a)s e colegas de classe comentam entre } \\
\text { si sobre seus pesos? }\end{array}$ \\
\hline $\begin{array}{l}\text { 34. How often do your friends and classmates encourage each other } \\
\text { to lose weight? }\end{array}$ & $\begin{array}{l}\text { 34. Com que freqüência seus amigo(a)s e colegas de classe encorajam um ao } \\
\text { outro a perder peso? }\end{array}$ \\
\hline $\begin{array}{l}\text { 35. How often do your friends and classmates talk about weight or } \\
\text { dieting? }\end{array}$ & $\begin{array}{l}\text { 35. Com que freqüência seus amigo(a)s e colegas de classe conversam sobre } \\
\text { peso ou prática de dietas? }\end{array}$ \\
\hline $\begin{array}{l}\text { 36. How often do your friends and classmates worry about their } \\
\text { weight? }\end{array}$ & $\begin{array}{l}\text { 36. Com que freqüência seus amigo(a)s e colegas de classe se preocupam } \\
\text { sobre seus pesos? }\end{array}$ \\
\hline 37. How often do your friends and classmates diet? & 37. Com que freqüência seus amigo(a)s e colegas de classe fazem dietas? \\
\hline 38. How often do your friends and classmates snack? & 38. Com que freqüência seus amigo(a)s e colegas de classe pulam refeições? \\
\hline $\begin{array}{l}\text { 39. Do you think that your friends and classmates pay a lot of } \\
\text { attention to each other's body shape? }\end{array}$ & $\begin{array}{l}\text { 39. Você acha que seus amigo(a)s e colegas de classe reparam muito no peso } \\
\text { e formas corporais um do outro? }\end{array}$ \\
\hline
\end{tabular}

\section{Consistência interna}

O instrumento demonstrou valores do coeficiente alfa de Cronbach satisfatórios, superiores a 0,80 conforme observado na Tabela 4 . Para as questões que integram o conteúdo mídia ( 1 a 10), família (11 a 28) e amigos (29 a 39) os valores corresponderam a 0,$80 ; 0,85$ e 0,91 , respectivamente.

\section{Discussão}

Embora haja inúmeras estratégias para o processo da adaptação cultural, que vão desde a simples tradução por parte dos pesquisadores ao processo mais minucioso que abrange e enfatiza a necessidade de diferentes nuanças na adaptação cultural 12, estudos recentes comprovam a importância em se valorizar as etapas de operacionalização na adaptação do instrumento 14 . 
Tabela 4

Avaliação dos especialistas e da população alvo da Escala de Influência dos Três Fatores (mídia, família e amigos).

\begin{tabular}{|c|c|c|c|c|}
\hline \multirow[t]{3}{*}{ Questões } & \multicolumn{2}{|c|}{ Especialistas ( $N=10)$} & \multicolumn{2}{|c|}{ População alvo ( $\mathrm{N}=108)$} \\
\hline & $\begin{array}{l}\text { Grau de concordância } \\
\text { do conteúdo: } \\
\text { mídia *, família **, } \\
\text { amigos *** }\end{array}$ & Grau de compreensão & $\begin{array}{l}\text { Grau de compreensão } \\
\qquad(n=53)\end{array}$ & $\begin{array}{l}\text { Consistência interna } \\
\qquad(\mathrm{n}=55)\end{array}$ \\
\hline & $\%$ & $\begin{array}{c}\text { Média } \\
\text { (desvios-padrão) }\end{array}$ & $\begin{array}{c}\text { Média } \\
\text { (desvios-padrão) }\end{array}$ & $\alpha$ de Cronbach \\
\hline 1 & 100,0 & $4,9(0,3)$ & $4,9(0,4)$ & \\
\hline 2 & 100,0 & $4,9(0,3)$ & $4,9(0,1)$ & \\
\hline 3 & 100,0 & $4,9(03)$ & $4,7(08)$ & \\
\hline 4 & 100,0 & $4,8(0,6)$ & $4,8(0,6)$ & \\
\hline 5 & 100,0 & $5,0(0,0)$ & $4,6(1,1)$ & \\
\hline 6 & 100,0 & $5,0(0,0)$ & $4,8(0,8)$ & 0,8 \\
\hline 7 & 100,0 & $5,0(0,0)$ & $4,7(0,8)$ & \\
\hline 8 & 100,0 & $5,0(0,0)$ & $4,7(0,9)$ & \\
\hline 9 & 100,0 & $5,0(0,0)$ & $4,7(0,9)$ & \\
\hline 10 & 100,0 & $5,0(0,0)$ & $4,7(0,9)$ & \\
\hline 11 & 100,0 & $4,4(1,0)$ & $4,7(0,9)$ & \\
\hline 12 & 100,0 & $4,8(0,4)$ & $4,7(0,9)$ & \\
\hline 13 & 100,0 & $4,4(1,0)$ & $4,7(0,8)$ & \\
\hline 14 & 100,0 & $4,9(0,3)$ & $4,7(0,9)$ & \\
\hline 15 & 100,0 & $4,9(0,3)$ & $4,6(1,2)$ & \\
\hline 16 & 100,0 & $4,7(0,7)$ & $4,6(1,1)$ & \\
\hline 17 & 100,0 & $4,9(0,3)$ & $4,7(1,0)$ & 0,85 \\
\hline 18 & 100,0 & $4,9(0,3)$ & $4,7(0,9)$ & \\
\hline 19 & 100,0 & $4,7(0,3)$ & $4,6(1,0)$ & \\
\hline 20 & 100,0 & $5,0(0,0)$ & $4,8(0,8)$ & \\
\hline 21 & 100,0 & $4,8(0,7)$ & $4,7(1,0)$ & \\
\hline 22 & 100,0 & $4,8(0,7)$ & $4,8(1,0)$ & \\
\hline 23 & 100,0 & $4,7(0,7)$ & $4,7(1,0)$ & \\
\hline 24 & 100,0 & $4,6(0,7)$ & $4,7(1,0)$ & \\
\hline 25 & 100,0 & $4,9(0,3)$ & $4,7(1,0)$ & \\
\hline 26 & 100,0 & $4,4(0,9)$ & $4,7(1,0)$ & \\
\hline 27 & 100,0 & $4,8(0,7)$ & $4,7(1,0)$ & \\
\hline 28 & 100,0 & $5,0(0,0)$ & $4,7(0,8)$ & \\
\hline 29 & 100,0 & $4,6(0,9)$ & $4,7(0,9)$ & \\
\hline 30 & 100,0 & $4,9(0,3)$ & $4,9(0,4)$ & \\
\hline 31 & 100,0 & $4,6(0,7)$ & $4,8(0,8)$ & \\
\hline 32 & 100,0 & $4,9(0,3)$ & $4,8(0,8)$ & \\
\hline 33 & 100,0 & $5,0(0,0)$ & $4,8(0,8)$ & \\
\hline 34 & 100,0 & $4,7(0,7)$ & $4,9(0,4)$ & 0,91 \\
\hline 35 & 100,0 & $4,6(0,7)$ & $4,9(0,4)$ & \\
\hline 36 & 100,0 & $4,9(0,3)$ & $4,9(0,3)$ & \\
\hline 37 & 100,0 & $4,6(0,7)$ & $4,9(0,4)$ & \\
\hline 38 & 100,0 & $4,4(0,8)$ & $4,9(0,8)$ & \\
\hline 39 & 100,0 & $5,0(0,0)$ & $4,9(0,5)$ & \\
\hline Total & 100,0 & $4,9(0,3)$ & $4,9(0,7)$ & 0,93 \\
\hline
\end{tabular}

* Questões de 1 a 10;

** Questões de 11 a 28;

$\star \star \star$ Questões de 29 a 39. 
Desta forma garante-se a veracidade e a qualidade da informação coletada.

O presente estudo cumpre este fim, apresentando uma escala e descrevendo seu processo de adaptação transcultural.

A Escala de Influência dos Três Fatores - amigos, família e mídia - foi traduzida, adaptada e apresentou valores satisfatórios na validação, demonstrando clareza quanto aos conteúdos que a compõem. Revelou igualmente ser de fácil compreensão, tanto para os especialistas como para a população alvo e apresentou valores satisfatórios na análise de consistência interna.

No processo de tradução e adaptação foi necessária a adequação de duas palavras específicas - fitness e skip meals. De acordo com Jorge 16 o significado geral transcende a literalidade dos termos sendo necessário considerar os aspectos culturais da população alvo. Este cuidado foi tomado no presente estudo. Ainda foram necessárias adaptações com inserções de expressões, padronização de pronomes e tempos verbais, com o intuito de garantir a compreensão do conteúdo expresso na escala original. Sendo assim, zelou-se pela pertinência, adequação e aceitabilidade do estilo empregado em cada item analisado.

Na versão final do instrumento as questões que contemplam a mídia foram preservadas, de acordo com a versão original. No que se refere às questões relacionadas à família e aos amigos foram excluídas quatro questões por não expressaram claramente o conteúdo e por serem repetitivas.

No estudo original 7, os valores da consistência interna dos fatores (amigos, família e mídia) corresponderam a 0,$89 ; 0,88$ e 0,87 , respectivamente. Tais valores são muito próximos aos registrados no presente estudo.

$\mathrm{Na}$ análise de conteúdo a escala pôde comprovar ser pertinente ao seu propósito, ou seja, avaliar a influência sócio-cultural, comprovou sua clareza e compreensão dos itens e demonstrou boa relação interna dos mesmos.

Cabe ainda salientar que os transtornos alimentares e os distúrbios da imagem corporal estão certamente entre os problemas mais comuns e debilitantes presentes entre os jovens 2 . Dos transtornos alimentares, estima-se que $4 \%$ das jovens norte-americanas desenvolvem bulimia nervosa, com os possíveis desdobramentos para as múltiplas comorbidades, o que inclui depressão, uso abusivo de substâncias psicoativas, entre outros 17. Para os meninos esta realidade não é diferente, no entanto, há especificidades no curso, no desfecho e no comprometimento psicossocial 18.

Os dados populacionais disponíveis 19,20,21 atrelados à experiência clínica comprovam que esses transtornos têm prevalência significativa no Brasil 22. Para que se possa conhecer esta realidade, um dos primeiros passos é disponibilizar instrumentos válidos e reprodutíveis para futuras aplicações.

De acordo com Reichenheim \& Moraes 14 é de extrema importância para os estudos epidemiológicos zelar pela qualidade da informação, visto ser este um elo entre o conteúdo teórico e o dado que expressa a realidade empírica. Sendo assim, o rigor dispensado no processo de aferição deve ocupar um lugar de destaque nesta prática. Este cuidado foi tomado na presente pesquisa.

A Escala de Influência dos Três Fatores encontra-se pronta para novos estudos que possam avaliar suas condições psicométricas. Sendo assim, são necessárias análises de validade externa, equivalência de mensuração e reprodutibilidade, como também, após a confirmação de suas propriedades psicométricas valeria a sua aplicação envolvendo populações específicas, tais como estudantes do Ensino Médio de escolas privadas e públicas. 


\section{Resumo}

O objetivo do estudo foi traduzir e adaptar o instrumento Tripartite Influence Scale para o idioma português e avaliar sua validade de conteúdo e consistência interna. Envolveu seis etapas: (1) tradução; (2) re-tradução; (3) revisão técnica e avaliação semântica; (4) validação de conteúdo por profissionais da áreajuízes; (5) avaliação do instrumento por uma amostra da população alvo, por meio da avaliação do grau de compreensão e (6) análise da consistência interna pelo coeficiente alfa de Cronbach. As 43 questões foram traduzidas e adaptadas para o idioma português. A versão final constou de 39 itens apresentando validação de conteúdo para os três construtos (mídia, família e amigos), clareza e fácil compreensão e boa concordância - valores do coeficiente de Cronbach superiores 0,80 . O instrumento encontra-se traduzido e adaptado para o idioma português demonstrando bons resultados no processo de validação de conteúdo, compreensão verbal e consistência interna. São necessárias análises de validade externa, equivalência de mensuração e reprodutibilidade.

Imagem Corporal; Transtornos Alimentares; Adolescente

\section{Colaboradores}

M. A. Conti participou da elaboração do projeto, redação e discussão do artigo. F. Scagliusi contribuiu na elaboração do projeto, revisão das análises e do artigo. G. K. O. Queiroz colaborou na discussão do projeto e do artigo. N. Hearst participou da tradução, revisão e discussão do artigo. T. A. Cordás colaborou na revisão e discussão do artigo.

\section{Referências}

1. Berkman ND, Lohr KN, Bulik CM. Outcomes of eating disorders: a systematic review of the literature. Int J Eat Disord 2007; 40:293-309.

2. Thompson JK, Smolak L. Body image, eating disorders and obesity in youth: assessment, prevention and treatment. Washington DC: American Psychological Association; 2003.

3. Thompson JK, Stice E. Thin-ideal internalization: mounting evidence for a new risk factor for bodyimage disturbance and eating pathology. Curr Dir Psychol Sci 2001; 10:181-3.
4. Shisslak CM, Crago M. Risk and protective factors in the development of eating disorders. In: Thompson JK, Smolak L, editors. Body image, eating disorders and obesity in youth - Assessment, prevention and treatment. Washington DC: American Psychological Association; 2003. p. 103-25

5. Jacobi C, Hayward C, de Zwaan M, Kraemer HC, Agras WS. Coming to terms with risk factors for eating disorders: application of risk terminology and suggestions for a general taxonomy. Psychol Bull 2004; 130:19-65. 
6. Tiggemann M. Media influence on body image development. In: Cash T, Pruzinsky T, editors. Body image: a handbook of theory, research, and clinical practice. New York: The Guilford Press; 2004 p. 91-8.

7. Keery H, van den Berg P, Thompson JK. An evaluation of the the Tripartite Influence Model of body dissatisfaction and eating disturbance with adolescent girls. Body Image 2004; 1:237-51.

8. Thompson JK, Heinberg LJ, Altabe M, TantleffDunn S. Exacting beauty: theory, assessment, and treatment of body image disturbance. Washington DC: American Psychology Association; 1999.

9. van den Berg P,Thompson JK, Obremski-Brandon K, Coovert M. The tripartite influence model of body image and eating disturbance: a covariance structure modeling investigation testing the mediational role of appearance comparison. J Psychosom Res 2002; 53:1007-29.

10. Pasquali L. Princípios de elaboração de escalas psicológicas. In: Gorenstein C, Andrade LHSG, Zuardi AW, organizadores. Escalas de avaliação clínica em psiquiatria e psicofarmacologia. São Paulo: Lemos Editorial; 2000. p. 15-21.

11. Herdman M, Fox-Rushby J, Badia X. A model of equivalence in the cultural adaptation of HRQoL instruments: the universalist approach. Qual Life Res 1998; 7:323-35.

12. Moraes CL, Hasselmann MH, Reichenheim ME. Adaptação transcultural para o português do instrumento "Revised Conflict Tactics Scales (CTS2)" utilizado para identificar violência entre casais. Cad Saúde Pública 2002; 18:163-76.

13. Conti MA, Latorre MRDO, Slater B. Tradução, validade e reprodutibilidade da EEICA - Escala de Evaluación da Insatisfación Corporal para Adolescentes - no Brasil. Rev Saúde Pública 2009; 43: 515-24.

14. Reichenheim ME, Moraes CL. Operacionalização de adaptação transcultural de instrumentos de aferição usados em epidemiologia. Rev Saúde Pública 2007 ; 41:665-73.
15. Clark P, Lavielle P, Martinez H. Learnig from pain scales: patient perspective. J Rheumatol 2003; 30:1584-8.

16. Jorge MR. Adaptação transcultural de instrumentos de pesquisa em saúde mental. In: Gorestein C, Andrade LHSG, Zuardi AW, organizadores. Escalas de avaliação clínica em psiquiatria e psicofarmacologia. São Paulo: Lemos Editorial; 2000. p. 53-9.

17. Pomov C. Assessment of physical factors in eating disorders. In: Thompson JK, Smolak L, editors. Body image, eating disorders, and obesity in youth Washington DC: American Psychological Association; 2001. p. 171-92.

18. Woodside D, Garfinkel PE, Lin E, Goering P, Kaplan AS, Goldbloom DS, et al. Comparisons of men with full or partial eating disorders, men without eating disorders, and women with eating disorders in the community. Am J Psychiatry 2001; 158:570-4

19. Nunes MA, Barros FC, Olinto MTA, Camey S, Mari JD. Prevalence of abnormal eating behaviours and inappropriate methods of weight control in young women from Brazil: a population-based study. Eat Weight Disord 2003; 8:100-6.

20. Ferreira JES, Veiga GV. Eating disorder risk behavior in Brazilian adolescents from low socio-economic level. Appetite 2008; 51:249-55.

21. Alves E, Vasconcelos FAG, Calvo MCM, Neves J. Prevalência de sintomas de anorexia nervosa e insatisfação com a imagem corporal em adolescentes do sexo feminino do Município de Florianópolis, Santa Catarina, Brasil. Cad Saúde Pública 2008; 24:503-12.

22. Pinzon V, Gonzaga AP, Cobelo A, Labaddia E, Belluzzo P, Fleitlich-Bilyk B. Peculiaridades do tratamento da anorexia e da bulimia nervosa na adolescência: a experiência do PROTAD. Rev Psiquiatr Clín 2004; 31:167-9.

Recebido em 29/Mai/2009

Versão final reapresentada em 18/Dez/2009

Aprovado em 04/Jan/2010 